\title{
Medical students' experience of a Teddy Bear Hospital as part of a paediatric curriculum
}

\author{
L. Nheu ${ }^{1}$, W. Uahwatanasakul, \& A. Gray ${ }^{1,2}$
}

\begin{abstract}
Introduction: Teddy Bear Hospital is a strategy used to help reduce children's fears of the healthcare system and teach them about health-related issues. It involves mock consultations where medical students play the role of a "teddy doctor" and children act as the carer of teddy, or another soft toy, requiring consultation. This provides medical students with the opportunity to interact with children and develop communication skills while children are introduced to the medical consultation process. The aim of this study was to evaluate the medical student experience of Teddy Bear Hospital as part of a paediatric curriculum.

Methods: We conducted a qualitative observational study using observations and focus group interviews with third-year postgraduate medical students. Strategies for engaging children and medical student behaviours were observed. In focus groups, students discussed their experience and the benefits of involvement they perceive. Inductive content analysis was performed, guided by a phenomenological approach, to identify themes.

Results: Three major themes were identified: student discomfort, adapting communication to child temperament and developmental age and how the Teddy Bear Hospital context impacts learning. Students experienced discomfort approaching families and through disclosure of medical information. Despite this, students described it as an enjoyable learning experience that taught them to adapt communication to the child. Students also recognised greater challenges in a hospital-based event than one in the community.
\end{abstract}

1 Department of Paediatrics, The University of Melbourne, Australia

2 Royal Children's Hospital, Melbourne, Australia

\section{Correspondence}

Wonie Uahwatanasakul

Department of Paediatrics, The University of Melbourne

Royal Children's Hospital

50 Flemington Road

Parkville, Victoria 3052

Australia

Tel: +61 393451445

Email: wonie.uahwatanasakul@rch.org.au 
Conclusions: Participation in Teddy Bear Hospital appears to be a valuable component of the paediatric curriculum for medical students. Through enjoyable experiential learning, students described developing important communication skills with children. While they did experience discomfort in the process, this has potential to deepen learning.

Keywords: medical education; Teddy Bear Hospital, medical students; paediatrics; learning; communication skills.

\section{Introduction}

Communication is a fundamental skill required in the medical profession (Noble \& Richardson, 2006). Good communication skills in health practitioners are associated with improved quality of life and symptom improvement, increased adherence to treatment plans, better management of chronic conditions in their patients and increased physician satisfaction with patient encounters, with reduced risk of medical errors and malpractice claims (Kurtz, 2002; Rider, Volkan, \& Hafler, 2008).

Communication skills can be taught to and learnt by medical students (Iqbal, Mookkappan, Basheer, \& Kandasamy, 2015; Smith et al., 2007; von Lengerke, Kursch, Lange, \& APG-Lehrteam, 2011) and are a core component of medical curricula. Interacting with children requires a different set of skills because of children's evolving cognitive abilities, emotional development and the dependent role of the parent and family in the decision-making process (Dube, LaMonica, Boyle, Fuller, \& Burkholder, 2003). These considerations, in combination with medical students often having minimal prior experience with children, increase students' discomfort interacting with children (Soares, Stratton, \& Wilson, 2015). Lack of confidence in paediatric communication skills has also been reported by residents (Rider et al., 2008).

Teddy Bear Hospital involves healthcare students role playing mock consultations with children, using soft toys that present with a variety of ailments (imagined and indicated by the child). Studies have shown that Teddy Bear Hospital reduces children's anxiety associated with hospitalisation and enhances their knowledge of the body, health and illness concepts as well as medical procedures (Bloch \& Toker, 2008; Leonhardt, Margraf-Stiksrud, Badners, Szerencsi, \& Maier, 2014).

There are numerous Teddy Bear Hospitals established worldwide, which have been implemented in various ways. Although a dominant aim of Teddy Bear Hospital is to help reduce children's anxiety associated with healthcare, there are presumed benefits for medical students as well. Previous descriptions of Teddy Bear Hospital programs have emphasised the potential benefit for students through volunteering in community service (Santen \& Feldman, 1994; Zimmermann \& Santen, 1997). Others have recognised the benefit for developing communication skills and acquiring knowledge about the growth and development of paediatric patients (Campbell \& Brown, 2008; Husøy, 2013). Medical student participation in Teddy Bear Hospital has also been described in the literature but without any formal evaluation of its impact (Friedman, 2014; Kaufman, Modak, \& Moylan, 2012). We aimed to evaluate the medical student experience of Teddy Bear Hospital as part of a paediatric curriculum in order to inform 
MEDICAL STUDENTS' EXPERIENCE OF A TEDDY BEAR HOSPITAL

its use as a medical education tool. In particular, we wanted to focus on perceived benefits for student learning in relation to communication, rapport building and child development, as well as the challenges they face.

\section{Teddy Bear Hospital program}

In our setting, Teddy Bear Hospital has been implemented in a number of contexts over time. Initially, volunteer students made planned visits to primary schools where children would participate in mock teddy bear consultations and receive health education through medical student run stations on basic anatomy, healthy foods and exercise. Teddy Bear Hospital became a charitable event in 2012, with over 500 health science students volunteering at an annual event for our hospital.

In 2014, the Teddy Bear Hospital program was first implemented in our hospital as a formal part of the Child and Adolescent Health medical curriculum, after a pilot program in 2013. The learning objectives of the session are to establish rapport with a child and his/her family in a manner in which the child and family feel comfortable, to converse with preschool and primary school-aged children using appropriate language and to explain a simple plan to a child.

Third-year postgraduate medical students at the hospital participate in Teddy Bear Hospital as part of their paediatric rotation. Medical students have had one year of clinical experience with rotations in adult tertiary-centred care facilities. The third year consists of rotations in paediatrics, women's health, aged care, psychiatry and general practice. Depending on the order of rotations and personal experience, medical students may or may not have had experience interacting with children prior to participating in Teddy Bear Hospital.

Participation by medical students in Teddy Bear Hospital is a compulsory part of our paediatric rotation. Teddy Bear Hospital is set up in the main hospital foyer, and groups of 8 to 10 medical students participate in a 2-hour session. Children and families who are in the hospital for other reasons are invited to attend if they walk through the foyer. Children may bring their own soft toy, though most borrow one of the hospital teddy bears for the purpose of the mock consultation. Medical students are also provided with basic materials, including bandages, syringes, tongue depressors, teddy $\mathrm{x}$-rays and teddy record booklets to facilitate the role plays. Sessions are held at the beginning of the paediatric rotation, after a week of introductory lectures. Each session is facilitated by a paediatrician to assist students interact with and approach children and their families and to invite them to participate in Teddy Bear Hospital. Students receive an introduction to Teddy Bear Hospital prior to participation and are provided with strategies and approaches to communicate with children. A debrief with the paediatrician facilitator occurs at the end of the session. 
MEDICAL STUDENTS' EXPERIENCE OF A TEDDY BEAR HOSPITAL

\section{Methods}

We conducted a qualitative observational study to evaluate how medical students experience Teddy Bear Hospital within the paediatric curriculum. Research was guided by a phenomenological approach, which aims to examine "the lived experience" of a person or several people in relation to a concept or phenomenon of interest (Liamputtong, 2009). We used two methods of data collection that were designed to be complimentaryobservation of Teddy Bear Hospital sessions and focus-group interviews. The use of observations and focus-group interviews allows for both methodological and source triangulation to ensure rigour (Liamputtong, 2009).

The primary researcher explained the project to medical students during the first week of their paediatric rotation, a week prior to the Teddy Bear Hospital. Participants received information sheets regarding observation of Teddy Bear Hospital sessions and focusgroup interviews. Participation in the study was voluntary, and participants gave informed consent by signing a consent form.

Observations and focus-group interviews with medical students were conducted by the primary researcher between February and April 2016, with two different cohorts of medical students completing their paediatric rotations at a paediatric teaching hospital. Ninety-eight medical students completed their paediatric rotation at the hospital over this period.

\section{Teddy Bear Hospital observations}

Eight sessions with 6 to 12 medical students $(n=61)$ were observed by the primary researcher. A pre-prepared template to guide observations was used (Figure 1). Studentchild interactions were observed, with attention paid to strategies used to engage children, language used by medical students, observed comfort of students and changes in comfort during the 2-hour session. Detailed field notes were recorded on paper forms and then converted to electronic format.

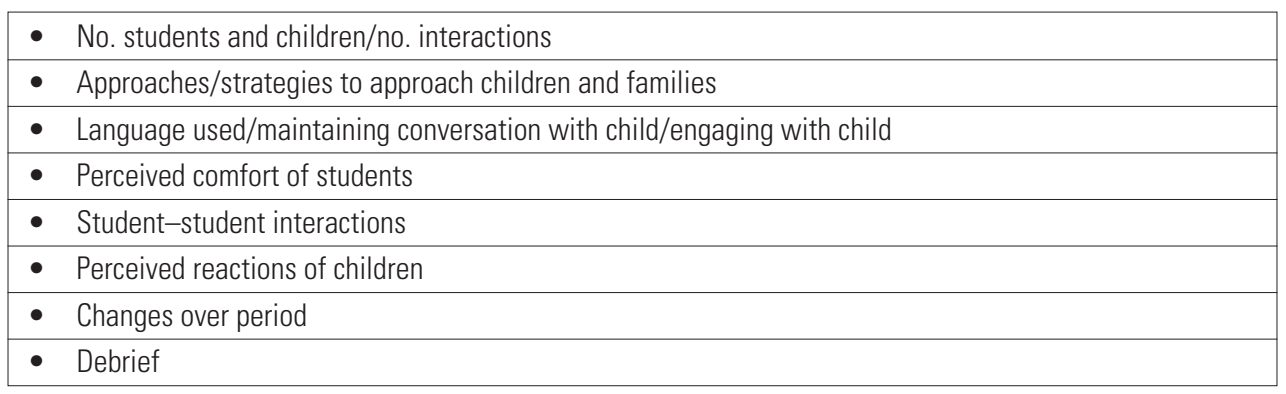

Figure 1. Template to guide observations. 
MEDICAL STUDENTS' EXPERIENCE OF A TEDDY BEAR HOSPITAL

\section{Focus-group interviews}

We conducted nine focus groups comprising four to eight medical students of mixed gender $(n=54)$. Six focus groups were conducted with the first cohort of medical students completing their paediatric rotation at the hospital (20 females, 17 males) and three with the second cohort (10 females, 7 males). Focus groups were held approximately 1 week after the Teddy Bear Hospital session. Focus groups were conducted using a semi-structured interview guide that explored aspects of the medical students' experience of Teddy Bear Hospital, including the perceived benefits and skills gained, difficulties encountered and suggestions for further improvement to the paediatric curriculum (Figure 2). All focus groups were audiotaped and transcribed verbatim.

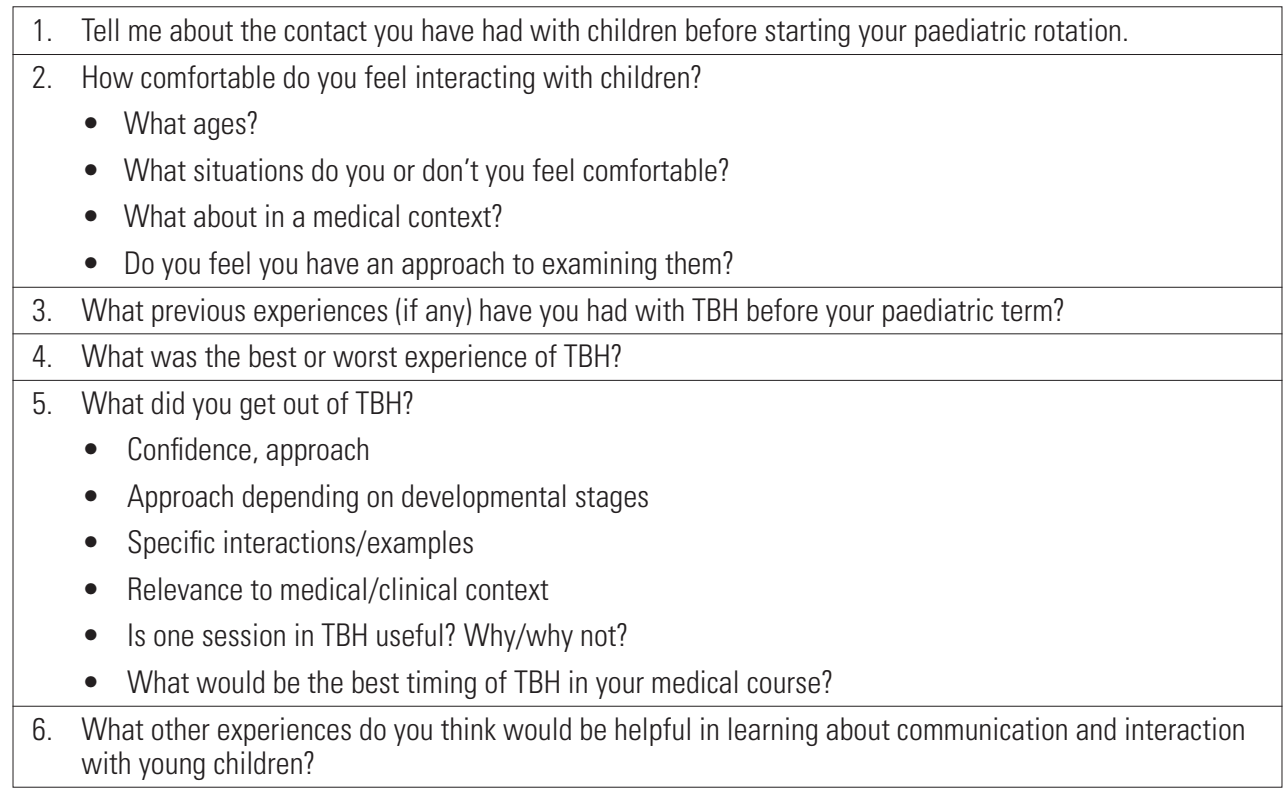

Figure 2. Interview guide for focus groups.

\section{Data storage and analysis}

Data from observations and focus groups were stored electronically on a passwordprotected computer accessible only by the primary researcher. Audiotaped files were deleted once transcribed.

Inductive content analysis was performed on transcripts of focus groups and field notes of observations using NVivo for Windows 10 to organise data. Transcripts were coded 
MEDICAL STUDENTS' EXPERIENCE OF A TEDDY BEAR HOSPITAL

separately by two researchers, who compared the codes and themes identified. Transcripts and codes were reviewed in an iterative process before settling on identified final themes to ensure rigour (Saldana, 2008; Tong, Sainsbury, \& Craig, 2007). Observations were used to complement and support findings of focus groups.

Ethical approval was obtained from the Royal Children's Hospital Human Research Ethics Committee in 2016 (HREC35280A).

\section{Results}

Analysis of data revealed three major themes:

- Student discomfort

- Adapting communication to developmental age and child temperament

- How the Teddy Bear Hospital context impacts learning

\section{Student discomfort}

Students described experiencing discomfort during the Teddy Bear Hospital session for a number of reasons. Firstly, students perceived an unclear boundary between being medical students with a purpose to learn and their role as representatives of the healthcare system, needing to conduct themselves accordingly. Students reported discomfort inviting children and families to participate in Teddy Bear Hospital. This was also observed by the primary researcher. Students appeared reluctant to approach children and families and at times required prompting by the facilitator of the session.

It is challenging because it's hard to build up confidence ... you're not sure if they're interested; you're not sure how they're going to react and interact with you. That's probably one of the more confronting things. (Focus group 8 , student 3)

There is that restraint ... carrying the fact that you're still meant to be professional. ... I think when you're in a hospital environment, it's obvious in our role as medical students. ... You try and toe that line between being professional and still being silly enough to get the kid engaged - it was a bit harder. (Focus group 8, student 5)

You're a lot more conscious of ... the negative impact that you could have on them and that parent. (Focus group 5, student 1)

Students felt out of their comfort zone managing difficult interactions. Interactions were made difficult by the disclosure of sensitive information, such as personal or medical problems and students feeling ill-equipped to respond. Other difficult interactions involved misbehaving children and, in some cases, difficulty closing an interaction.

And she [the parent] was like "oh, he's not doing great." I was like "I don't know what you want me to talk about, without crossing the line". So that was a bit hard. (Focus group 1, student 1)

It went far beyond any consultation I would usually give to any patient. I didn't know how to, at what point it was appropriate to end it. (Focus group 6, student 3) 
MEDICAL STUDENTS' EXPERIENCE OF A TEDDY BEAR HOSPITAL

Finally, students reported feeling awkward, nervous and anxious about interacting with children, particularly those with less prior experience. This discomfort was observed in some students, particularly at the beginning of the Teddy Bear Hospital session. However, this was not true for all students, and a proportion were quite comfortable approaching and interacting with children from the outset.

I find that sometimes I'm a bit awkward with children and not having had that experience before. (Focus group 8, student 1)

I was so anxious about it. ... I was freaking out. (Focus group 1, student 6)

It was not a huge challenge for me because I'm quite an outgoing individual. (Focus group 8, student 3)

Despite significant discomfort, most students reported an increase in confidence, gained during the Teddy Bear Hospital session. This correlated with observations of student interactions made by the primary researcher. As students became more comfortable, we observed increased interaction time, increased one-on-one interactions with children and greater initiative taken in approaching children to participate.

It got easier over time. (Focus group 1, student 6)

I'm more confident in my interactions with kids now. ... Before I wasn't sure. (Focus group 4, student 6)

It was a bit awkward and slow at the start, but by the end, I had gotten into the swing of things, and it was a really fun experience, quite enjoyable. (Focus group 3, student 3)

\section{Adapting communication to developmental age and child temperament}

Students identified several skills gained or developed during participation in Teddy Bear Hospital. Approaches such as being friendly and taking advantage of equipment available were identified as helpful in engaging with children. Students learned to use teaching as a tool to engage older children. During observations, it was noted that students adapted methods of engaging children depending on the age of the child. Younger children were more receptive to imaginative role play, while older children responded well to learning about anatomy.

Older kids found ... the challenge of figuring out what all the organs do quite interesting. ... The younger kids didn't really engage with this, but the older kids found it quite interesting. (Focus group 3, student 3)

Students recognised the value of using others, such as their peers or the parents and siblings of the child, to facilitate communication with children and were observed doing this by the researcher.

It was good to sort of test the waters ... as to how much you could get out of a five-year-old as opposed to ... a two-year-old, and ... change your communication with them to suit their age. I thought it was really helpful for that because I was completely pitching it at the wrong level. (Focus group 6, student 2) 
MEDICAL STUDENTS' EXPERIENCE OF A TEDDY BEAR HOSPITAL

There were quite a few shy kids that came through, and it was engaging with the parents that really helped there. (Focus group 9, student 2)

Students appreciated the wide variety of children they encountered during the Teddy Bear Hospital session, which enabled them to observe differences in children depending on age, developmental stage, temperament and health status. Each of these differences generated difficulties in interactions with children. Difficulties included choosing appropriate language and tone, interacting with late primary-school-aged children (8-12 years) and recognising the developmental stage of children with delay.

That's the good thing about Teddy Bear Hospital ... you see that every child is completely different. (Focus group 2, student 5)

There was also that aspect of developmental delay. And that was something that was hard to account for. (Focus group 7, student 2)

Differences in child temperament were perceived as a major contributor to the ease or difficulty of a given interaction. Shy children were harder to engage, with many strategies required, but it was rewarding once a child came "out of their shell".

One of the most satisfying things ... that moment when they decide "yeah, I'll actually do this" is quite rewarding and makes you feel like you're getting somewhere and that you're able to do it. (Focus group 5, student 1)

\section{How the Teddy Bear Hospital context impacts learning}

Students reported Teddy Bear Hospital was an enjoyable learning experience, providing a good introduction to paediatrics. However, students also recognised that hospital-based Teddy Bear Hospitals provided different learning opportunities and challenges to those run in schools or at charitable events. These learning opportunities related to children who had chronic illnesses or developmental problems attending Teddy Bear Hospital. These additional challenges may have heightened the sense of discomfort students experienced in our hospital-based Teddy Bear Hospital.

Despite this, students were able to practice communication skills outside of the clinical context, through experiential learning, providing a basis for skills to be further developed throughout their paediatric rotation.

An introductory session to interacting with children and then throughout your whole paediatric term. ... It builds on top of that. (Focus group 9, student 1)

I really enjoyed it. ... I think it's a very useful thing for medical students to do. I think it's kind of good to have that interaction, outside of the medical context, where you don't have to think about... all the stuff you're meant to be thinking about, and just sort of focus on the interaction. (Focus group 1, student 8)

Students identified similarities to previous Teddy Bear Hospital experiences, in particular being involved in the community in the context of a charitable event. However, a large number of students reported differences in Teddy Bear Hospital outside of the hospital setting, where participation is voluntary (for the medical student). The first difference 
identified was the population of children seen, as children were more likely to have chronic health problems or developmental issues in the hospital setting. Secondly, students needed to approach children and families who were at the hospital for various reasons and were not expecting to participate in Teddy Bear Hospital on that day. These differences made interactions more difficult for students.

Obviously it was a bit different. ... Some of the kids themselves were actually sick, whereas the Teddy Bear Hospital at [the charitable event], a lot of them were well children. So I found a lot of the kids were kind of projecting their own illness... onto their teddy bears. (Focus group 5, student 4)

I think the ones outside the hospital ... they're somewhat easier, because the children know that they're coming there. ... Whereas, I think the ones at the hospital are a bit harder. ... It's a bit more challenging to try and get the kids involved. (Focus group 8, student 2)

\section{Discussion}

Through role play in Teddy Bear Hospital, medical students developed communication skills and gained confidence interacting with children. This supports similar studies conducted involving nursing students (Campbell \& Brown, 2008; Husøy, 2013). Previous publications have provided a program description of Teddy Bear Hospital and the presumed benefits for medical students (Friedman, 2014; Kaufman et al., 2012). Our study evaluated the actual experience of medical students and correlated this with observations made at the time by one of the researchers. We found that Teddy Bear Hospital is perceived to be a valuable tool in the medical curriculum, providing an enjoyable learning experience in which students are introduced to paediatrics. Our Teddy Bear Hospitals provided many challenges for medical students from which specific learnings were gained, both personal and practical. Initial discomfort and anxiety associated with interacting with children gave way to an increase in confidence during participation in Teddy Bear Hospital. The ability to interact with a wide range of children provided medical students with the opportunity to develop, apply and practise communication skills. We hope that these skills are transferable to the clinical setting, though this warrants further study.

The findings in this study support Teddy Bear Hospital as a learning tool within the medical curriculum. Learning and developing communication skills through delivering health education programs to children has been recognised (Begley, Haddad, Christensen, \& Lust, 2009; Olm-Shipman, Reed, \& Christian, 2003; Stefaniak \& Lucia, 2014), and Teddy Bear Hospital may be seen as one such program. The program also shares similarities with programs where kindergarten visits are used to provide experience for medical students with younger children (McConnell et al., 2010; Pham, Chan, Williams, Zwi, \& White, 2010).

A difference, however, is in the population of children seen by our medical students. In Teddy Bear Hospital, there is a wider range of children seen, in age and health status. The children participating in our Teddy Bear Hospitals are not necessarily "well" children, as many are present for outpatient appointments or may be inpatients themselves. Although this increases the difficulty for students, putting students into this "zone of discomfort" 
potentially leads to greater experiential learning and more authentic learning outcomes. The difficulty described in managing interactions has parallels to those encountered when consulting patients and their families in clinical settings, for example, initiating consultations and being clear about their purpose, being asked questions for which you may not have the answer or not be the right person to answer and ending the consultation without the family feeling like they are cut off. Although recognised by students as one of the challenges of Teddy Bear Hospital in our setting, most did not relate managing these interactions as a learning outcome relevant to the clinical context. Increasing student awareness of the skills required to manage patient interactions, beyond specific communication skills, may add more value to the Teddy Bear Hospital program.

A strength of this study lies in the large proportion of medical students that took part in this research, and therefore, we believe our results are likely to be representative of the local medical student cohort as a whole, with a wide range of experiences and perspectives represented. The study also has limitations, including the use of only one observer during data collection. Useful interactions between medical students and children in Teddy Bear Hospital may have been missed. Interactions may have also been impacted by having an observer present. There are also potential limitations with focus-group interviews since students who have positive experiences are more likely to participate, potentially skewing the perspectives in the data. Some participants expressing strong opinions may have discouraged others from providing opposing views. Differences in group dynamic may have also contributed to the direction and quality of discussions between each focus group.

Further work is needed to understand how the learning outcomes identified here are translated into student communication skills in a clinical paediatric setting and, ultimately, to student-patient interaction. There is also potential to use quantitative survey methods to explore the relevance of our findings across a larger student cohort or to compare the impact of Teddy Bear Hospital in different settings.

\section{Conclusion}

Our study supports the value of Teddy Bear Hospital in the paediatric curriculum for medical students. This is important, not in the least because delivery of the program requires substantial investment in organisation, resources and facilitation. Through an enjoyable learning experience, medical students develop important communication skills and appreciate the challenges of communication with children with a range of developmental ages, temperaments and states of health. There is great potential to translate these skills to the clinical context, yet students did not recognise this relationship. Students also experienced some discomfort learning in this setting and, thus, adequate preparation and debriefing is required when planning a Teddy Bear Hospital in a clinical environment. This program may serve as an example for other medical or health-student curricula. The results of this evaluation reaffirm the value of health education programs as a means of teaching medical students. 
MEDICAL STUDENTS' EXPERIENCE OF A TEDDY BEAR HOSPITAL

\section{Acknowledgements}

The authors would like to thank the medical students who took part in the study and Dr Jan Hodgson for her support in qualitative research methods.

\section{Funding and conflicts of interest}

There are no conflicts of interest to declare. Teddy Bear Hospital is conducted as part of the University of Melbourne medical student curriculum and no additional funding was required for this study.

\section{References}

Begley, K., Haddad, A. R., Christensen, C., \& Lust, E. (2009). A health education program for underserved community youth led by health professions students. American Journal of Pharmaceutical Education, 73(6), 98.

Bloch, Y. H., \& Toker, A. (2008). Doctor, is my teddy bear okay? The "Teddy Bear Hospital" as a method to reduce children's fear of hospitalization. Israel Medical Association Journal, 10(8-9), 597-599.

Campbell, A., \& Brown, S. T. (2008). The Healthy Teddy Clinic: An innovative pediatric clinical experience. Teaching and Learning in Nursing, 3(2), 72-75.

Dube, C. E., LaMonica, A., Boyle, W., Fuller, B., \& Burkholder, G. J. (2003). Selfassessment of communication skills preparedness: Adult versus pediatric skills. Ambulatory Pediatrics, 3(3), 137-141.

Friedman, D. M. (2014). Alpert medical students dispel fears at Teddy Bear Clinic. Rhode Island Medical Journal, 97(1), 60-61.

Husøy, G. (2013). Teddy Bear Hospital: Students' learning in the field of practice with children. Nordic Journal of Nursing Research, 33(1), 51-55.

Iqbal, N., Mookkappan, S., Basheer, A., \& Kandasamy, R. (2015). Impact of focused training on communication skills of final-year medical students in a medical school in India. Australasian Medical Journal, 8(10), 325-332.

Kaufman, J., Modak, M., \& Moylan, S. (2012). The Teddy Bear Hospital in Australia. Journal of Paediatrics and Child Health, 48(6), 541-542.

Kurtz, S. M. (2002). Doctor-patient communication: Principles and practices. Canadian Journal of Neurological Sciences, 29(Suppl. 2), S23-S29.

Leonhardt, C., Margraf-Stiksrud, J., Badners, L., Szerencsi, A., \& Maier, R. F. (2014). Does the "Teddy Bear Hospital" enhance preschool children's knowledge? A pilot study with a pre/post-case control design in Germany. Journal of Health Psychology, 19 (10), 1250-1260.

Liamputtong, P. (2009). Qualitative research methods. Oxford, England: Oxford University Press. 
MEDICAL STUDENTS' EXPERIENCE OF A TEDDY BEAR HOSPITAL

McConnell, E., Clasen, C., Stolfi, A., Anderson, D., Markert, R. J., \& Jaballas, E. (2010). Community service and the pediatric exam: An introduction to clinical medicine via a partnership between first year medical students and a community elementary school. Teaching and Learning in Medicine, 22(3), 187-190.

Noble, L., \& Richardson, J. (2006). Communication skills teaching: Current needs. The Clinical Teacher, 3(1), 23-28.

Olm-Shipman, C., Reed, V., \& Christian, J. G. (2003). Teaching children about health, part II: The effect of an academic-community partnership on medical students' communication skills. Education for Health, 16(3), 339-347.

Pham, M., Chan, B., Williams, K., Zwi, K., \& White, L. (2010). Introducing clinical paediatrics to medical students: A novel hospital visitation programme involving kindergarten children. Medical Teacher, 32(7), e276-e281.

Rider, E. A., Volkan, K., \& Hafler, J. P. (2008). Pediatric residents' perceptions of communication competencies: Implications for teaching. Medical Teacher, 30(7), e208-e217.

Saldana, J. (2008). Coding manual for qualitative researchers. Los Angeles, CA: Sage.

Santen, L., \& Feldman, T. (1994). Teddy bear clinics: A huge community project. MCN: The American Journal of Maternal Child Nursing, 19(2), 102-106.

Smith, S., Hanson, J. L., Tewksbury, L. R., Christy, C., Talib, N. J., Harris, M. A., ... Wolf, F. M. (2007). Teaching patient communication skills to medical students: A review of randomized controlled trials. Evaluation \& the Health Professions, 30(1), 3-21.

Soares, N., Stratton, T., \& Wilson, J. (2015). Medical students' comfort with children. The Clinical Teacher, 12(3), 176-180.

Stefaniak, J. E., \& Lucia, V. C. (2014). Physician as teacher: Promoting health and wellness among elementary school students. Education for Health, 27(2), 183-187.

Tong, A., Sainsbury, P., \& Craig, J. (2007). Consolidated criteria for reporting qualitative research (COREQ): A 32-item checklist for interviews and focus groups. International Journal for Quality in Health Care, 19(6), 349-357.

von Lengerke, T., Kursch, A., Lange, K., \& APG-Lehrteam, M. M. H. (2011). The communication skills course for second year medical students at Hannover Medical School: An evaluation study based on students' self-assessments. GMS Journal for Medical Education, 28(4), Doc54. doi:10.3205/zma000766

Zimmermann, P. G., \& Santen, L. (1997). Teddy says "Hi!": Teddy bear clinics revisited. Journal of Emergency Nursing, 23(1), 41-44. 